\title{
Switching Cell of Directional Coupler Embedded in Photonic Crystal Driven by an External Command (for the C, L, and U bands of ITU)
}

\author{
Amir Wirth Lima Junior. and Antonio Sergio Bezerra Sombra
}

\begin{abstract}
We analyze and propose a directional optical coupler embedded in photonic crystal (PhC), which is driven by an external command signal. The switching method uses a low power external command signal, inserted in the central coupling region, which acts as another waveguide. The switching process is based on the change of the coupler from the bar state to the cross state owing to the external command signal.
\end{abstract}

Index Terms - Coupling Region, Directional Coupler, Photonic Crystal, Switching Cell.

\section{INTRODUCTION}

$\mathrm{D}$ UE to the nanometer dimension of the periodicity found in photonic crystals (PhCs), we can use directional coupler embedded in photonic crystal to act as switching cell within photonic integrated circuits (PICs).

Theoretical studies of basic directional coupler structure [1, 2] and improved structures e.g. a structure with shorter coupling length [3] and experimental study [4], have been done. However, the major obstacle to the use of such devices as switching cell is that the switching between the input and output ports needs to be activated by an external signal. Therefore, we propose a directional coupler embedded in photonic crystal driven by an external command signal. This command signal, which is strongly confined in the coupling region, increases the effective refractive index and consequently the coupling coefficient is reduced. Thus the coupler, which was designed to operate in the straight state can now works in the cross state. Taking into account that the power of the command signal for obtaining the needed difference in the refractive index to bring the coupler of the straight state to the cross state is a function of the Kerr coefficient, of the group velocity and of the coupler length, we can use these three parameters to determine the lowest possible power of the command signal. However, due to the great difficulty in obtaining the changing of the group velocity and of the Kerr coefficient, the most feasible is to increase the

Manuscript received October 9, 2008; revised November 27, 2008. This work was supported in part by National Council of Scientific and Technological Development (Conselho Nacional de Desenvolvimento Científico e Tecnológico- CNPq). Wirth Lima, A. J. Federal University of Ceará (corresponding author) phone: 55-85-30878964; e-mail: awljeng@ gmail.com. Sombra, A. S. B., Federal University of Ceará e-mail: sergio@fisica.ufc.br coupler length by the power periodic transfer characteristic of this device.

\section{THEORETICAL MODEL}

According to the supermodes method, in an optical coupler with two singlemode waveguides there are only two supermodes traveling in the structure, which possess different propagation constants $\left(\beta_{\text {even }}\right.$ and $\left.\beta_{\text {odd }}\right)$. Hence, the coupling length of a symmetrical directional coupler is

$L_{c}=\frac{\pi}{\beta_{\text {odd }}-\beta_{\text {even }}}($ Cross state $)$

From the coupled mode method, the coupling length of a symmetrical directional coupler is;

$L_{c}=\frac{\pi}{2 k}$ (Cross state)

Taking into account a low-intensity $\mathrm{CW}$ beam, if we neglect the SPM and XPM effects, the amplitude (A) and intensity (I) of the signal is function of the traveled distance within the coupler (z), and of the coupling coefficient, according to the following equations [5]:

$A_{a}(z)=A_{0} \cos (k z)$ and $I_{a}(z)=A_{0}^{2}(\cos (k z))^{2}$

$A_{b}(z)=-i A_{0} \sin (k z)$ and $I_{b}(z)=A_{0}{ }^{2}(\sin (k z))^{2}$

Hence, if $\mathrm{kz}=\pi / 2$, the optical power inserted into one of the two input ports is fully transferred to the exit port corresponding to the adjacent waveguide where the signal was inserted (cross state). On the other hand, if $\mathrm{kL}_{\mathrm{c}}=\pi$ the optical power inserted into one of the two input ports is fully transferred to the exit port corresponding to the same waveguide where the signal was inserted (bar state).

Therefore, if $z=L_{c}$, where $L_{c}$ is the coupling length we get $L_{c}=m \frac{\pi}{2 k}$.

Hence, when " $m$ " is odd the coupler is operating in the cross state. On the other hand, when " $\mathrm{m}$ " is even the coupler is operating in the bar state.

\section{Coupler Detlailing}

We present a directional coupler embedded in photonic crystal, which acts as switching cell. This coupler is embedded in a two-dimensional (2D) triangular lattice of dielectric rods 
[6]. The structure shown in figure 1 is introduced in order to obtain the dispersion relation, which is most appropriate for our switching cell.

The radiuses of the silicon rods of center and outside of waveguides are enlarged to $0.445 \mathrm{a}$ and $0.33 \mathrm{a}$, respectively. (The radiuses of the another rods $\left(\mathrm{SiO}_{2}\right)$ are $\left.0.29 \mathrm{a}\right)$. The position of the silicon rods of outside of waveguides are shifted $0.213 \mathrm{a}$ towards the center of the structure. The refractive index of the dielectric rods are $n=3.45$ (Silicon), which are embedded in a substrate with refractive index $\mathrm{n}_{\mathrm{s}}=$ $1.45\left(\mathrm{SiO}_{2}\right)$ [7].

The fabrication of the similar devices proceeds as follows. A $350 \mathrm{~nm}$ thick layer of ZEP-520A is spun onto a SOI wafer supplied by SOITEC $(220 \mathrm{~nm} \pm 5 \mathrm{~nm}$ thick Si layer, $2 \mu \mathrm{m}$ $\mathrm{SiO} 2$ buffer) to act as a resist and etch mask. The patterns were generated by electron beam lithography, and, following development of the resist, transferred directly into the Si layer using low-power, low-DC bias reactive ion etching in a CHF3/SF6 gas mix, a process known to yield very low-loss photonic crystal waveguides. A silica overlayer and the infilling of the etched structures is provided by a spin-on flowable oxide, which is hard-baked at $400^{\circ} \mathrm{C}$ for 3 hours [8].

Taking into account the study in two dimensions $\mathrm{PhC}$ (the $\mathrm{z}$ coordinate in the thickness direction is considered infinite, and is perpendicular to the plane $(\mathrm{x}, \mathrm{y})$, which is the region of propagation of the signals), we can consider that the wave within the PhC consists of two uncoupled modes: TE polarization (" $\mathrm{z}_{\text {even }}$ ") and TM polarization (" $\mathrm{z}_{\mathrm{odd}}$ "). In this case the TE mode is defined as having the polarization components of the electric field located only in the $(\mathrm{x}, \mathrm{y})$ plane, while polarization components of the magnetic field has only component in the direction $z$. Note that this definition of TE polarization is different from TE polarization set to conventional waveguide type, where the components of the electric field are located only in the transverse direction of propagation of the signal, while the magnetic field has component in the direction of propagation of the signal. The same reasoning can be applied to the methods considered to TM modes, which has polarization components of the magnetic field only at $(\mathrm{x}, \mathrm{y})$, while the electric field has polarization component in the $\mathrm{z}$ direction.

We use the PWE [9] and FDTD methods in the analysis described below. The bandgap of the feasibles modes is 0.283 $<\mathrm{u}<0.325(\mathrm{u}=$ normalized frequency $=\mathrm{a} / \lambda)$ for TM modes.

We are interested in finding the coupler to operate in bands $\mathrm{C}, \mathrm{L}$ and $\mathrm{U}$ of ITU (1530nm to $1675 \mathrm{~nm}$ ). As the wavelength $\lambda$ $=1.55 \mu \mathrm{m}$ is currently the most widely used in optical communications we chose $\mathrm{a}=450 \mathrm{~nm}$ to get $\mathrm{u}=0.29$ referring to this wavelength.

The signal switching is possible due to the different propagation constants of the two split modes that arise in the system [10].

Figure 2 shows the dispersion relation related to even and odd modes of the coupler, obtained by means of PWE. We can apply the equation (1) for each normalized frequency and thus obtain the required coupler length. For example, if we choose $\mathrm{u}=0.29$ we get $\mathrm{L}_{\mathrm{c}} \approx 326 \mu \mathrm{m}$, which is the minimal coupler length (bar state).

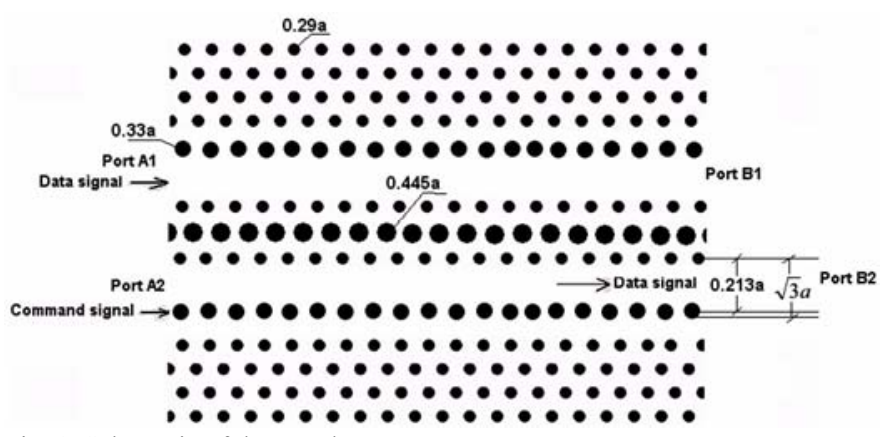

Fig. 1. Schematic of the coupler.

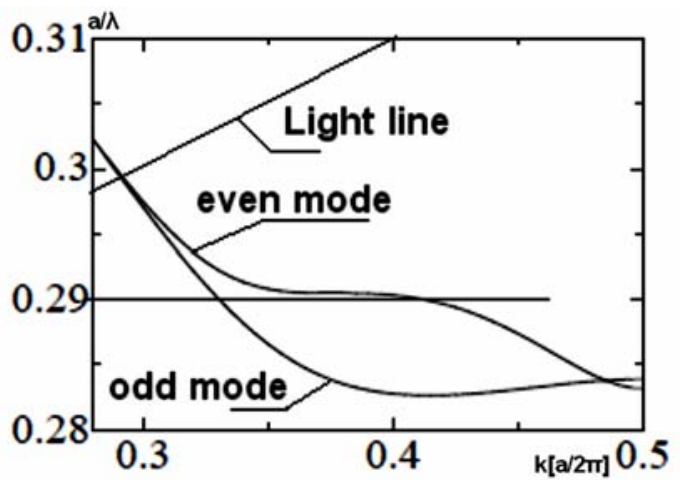

Fig. 2. Dispersion relation of the PhC coupler (even mode and odd mode).

The command signal holds frequency within the PBG, but outside of the $1530 \mathrm{~nm}-1675 \mathrm{~nm}$ band. This signal is inserted in the middle of the coupling region, which operates as a periodic waveguide [11].

Considering that the data and command signals are TM polarized, and that the signals used in telecommunications have randon polarization is necessary that these signals pass through a polarizer before entering the coupler.

Notice that we use only modes that lie below the light line, which are guided with no intrinsic losses (truly guided modes). Modes that lie above the light line have an intrinsic loss mechanism due to out-of-plane diffraction (quasi-guided modes).

If the command signal is working in the coupling region the refractive index increases (due to the non-linear effects), which causes the decrease of the coupling coefficient value (Please see details below). If we consider that the coupler was designed to operate in the bar state, the decrease of the coupling coefficient should be sufficient to bring the coupler to work in the cross state (Figure 3).

Figure 4 shows the dispersion relation of the command signal obtained by means of PWE. Note that the normalized frequency (u) band and wave vector $(\mathrm{k})$ band related to the supermodes dispersion relation is different from the normalized frequency band and wave vector band related to the command signal, respectively.

To get low optical power for the command signal, we need low value of $\Delta \mathrm{n}$, and/or high value of $\mathrm{n}_{2}$. For a polarization- 
preserving fiber with core made of pure silica $\mathrm{n}_{2}=2.66 \times 10^{-20}$ $\mathrm{m}^{2} / \mathrm{W}$. Nevertheless, the last experiments showed that we can adopt $\mathrm{n}_{2}=1.2 \times 10^{-17} \mathrm{~m}^{2} / \mathrm{W}(\lambda=1.8 \mu \mathrm{m})$. [12]. Thus, the normalized frequency of the command signal is $\mathrm{u}=0.25$.

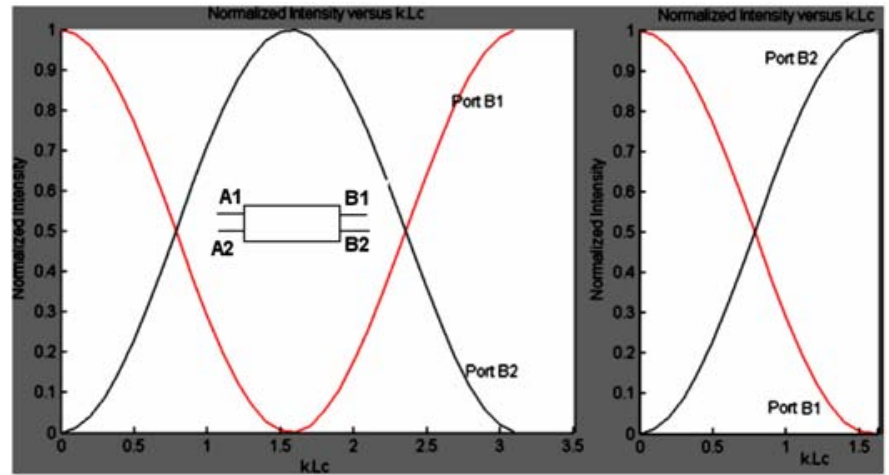

Fig. 3 (a) Prior to the command signal insertion, all entry optical power exits through the B1 port. (b) After the command signal insertion all entry optical power now exits through the B2 port.

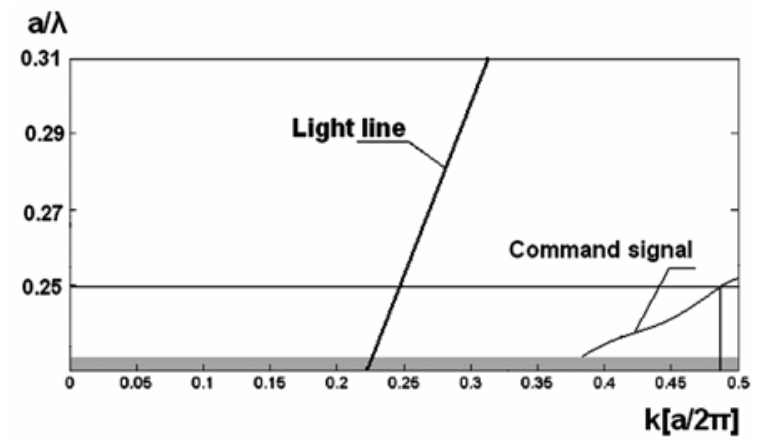

Fig. 4. Dispersion relation of the command signal.

The three signals inside the device could cause four-wave mixing. However, for this to occur would require the matching of the frequencies as well as of the wave vectors (phase-matching). The phase matching requirement is that $\Delta \mathrm{kj}$ $=0(\mathrm{kj}=\mathrm{njw} / \mathrm{c}), \mathrm{j}=1$ to 4 .

It is difficult to satisfy phase-matching in our device as result of variations in the structure of the PHC.

Figure 5(a) shows the field distribution concerning the data signal $(\mathrm{u}=0.29)$ inside the coupler with length of $2 \mathrm{xL}_{\mathrm{c}}$ (bar state) using the FDTD, before the insertion of the command signal, which was obtained by FDTD. At Figure 5(b) we show the field distribution of the data signal inside this coupler, after the insertion of the command signal (cross state).

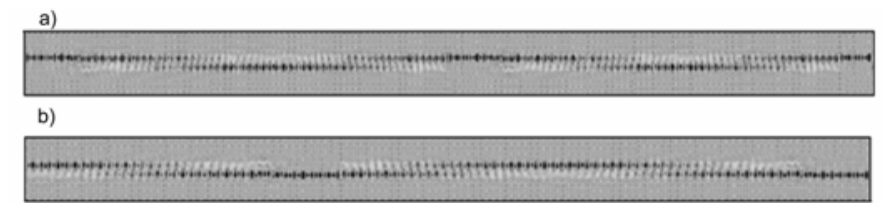

Fig. 5. (a): Field distribution concerning the data signal inside the coupler with length of $2 * \mathrm{Lc}$, before the insertion of the command signal. (b): Field distribution of the data signal inside the coupler, after the insertion of the command signal.

Figure 6 (obtained by COMSOL) shows the electric field distribution referring to the command signal inside the coupler.

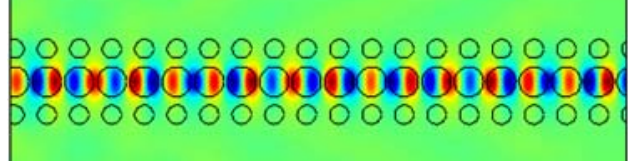

Fig.6 . Electric field distribution referring to the command signal inside the coupler.

We can notice that the field mode has most of its energy concentrated in the silicon rods.

The calculation of the necessary power should take into account the increase of the refractive index in the coupling region $(\Delta n)$, which depends on the used $\mathrm{PhC}$ structure, and on the normalized frequency of the command signal.

$$
P=\frac{(\Delta n) A_{\text {eff }}}{3 n_{2}\left(\frac{\left.v g\right|^{u}}{\left.v g\right|^{c}}\right)}
$$

In equation (5) [13] $\mathrm{P}$ is the desired optical power of the command signal, $\mathrm{n}_{2}$ is the non-linear refractive index, whose maximum value happens to the adopted wavelength around $1800 \mathrm{~nm}, \mathrm{~A}_{\text {eff }}$ is the mode effective area, $\left.v g\right|^{u}$, is the command signal group velocity in a conventional axial uniform waveguide, and $\left.v g\right|^{c}=0.03 * \mathrm{c},(\mathrm{u}=0.25)$ is the group velocity of the command signal in the coupling region. We consider in this equation the 2 factor (cross phase modulation (XPM) induces twice variance of the refractive index regarding the self-phase modulation (SPM)) and the 1.5 factor (the longitudinal mode confinement is not uniform). Moreover, we still consider the ratio of the group velocity in the center of a uniform conventional axial waveguide, regarding group velocity in the periodic waveguide.

Since our coupler, was originally designed to operate in the bar state, and we need modify the coupler to operate in the cross state;

$\Delta \beta_{c} L_{c}=\pi$. Taking into account that $\Delta \beta_{d} L_{c}=2 \pi$, then we get $\Delta \beta_{c}=\frac{\Delta \beta_{d}}{2}$. Hence, we must use the command signal to reduce the value of $\Delta \beta_{\mathrm{d}}$ to half of its original value, so that our coupler can change to the cross state.

However, for the coupler with length $L=2 x L c$ to change from the bar state to the cross state it is need that $\Delta \beta_{\mathrm{c}}(2) 2 \mathrm{~L}_{\mathrm{c}}=$ $3 \pi$, ie: $\Delta \beta_{c(2)}=\frac{3 \pi}{2 L_{c}}=\frac{3}{2} \frac{\Delta \beta_{d}}{2}=\frac{3}{4} \Delta \beta_{d}$.

This means that for $\mathrm{L}=\mathrm{nL}_{\mathrm{c}}$ we get

$\Delta \beta_{c(n)}=\frac{(2 n-1) \pi}{n L_{c}}=\frac{(2 n-1)}{2 . n} \Delta \beta_{d}$.

\section{Switching Cell Analysis}

Figure 7 shows the needed difference between the propagation constant of the modes $(\Delta \beta)$ for the switching of the bar state to the cross state regarding the possible lengths of our coupler to the wavelength $\lambda=1,55 \mu \mathrm{m}$. 


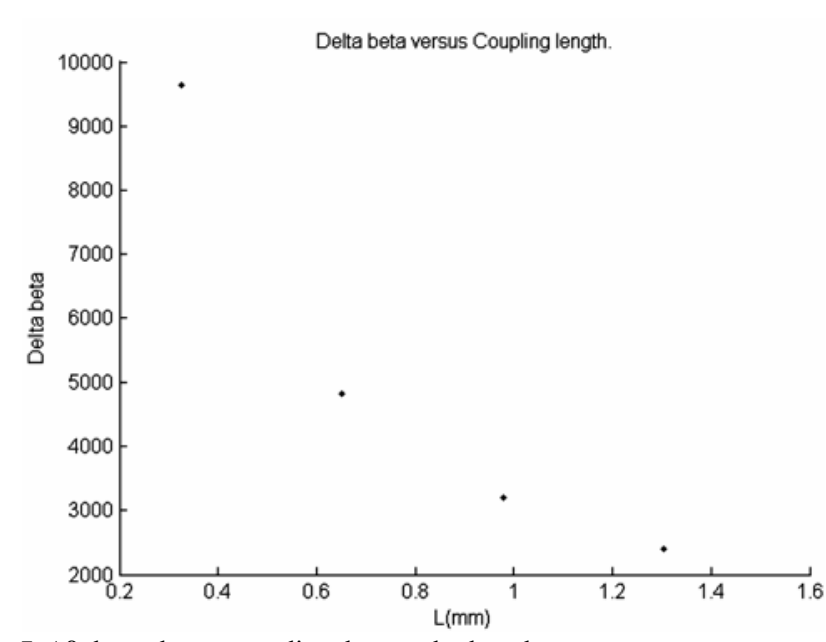

Fig. 7. $\Delta \beta$ dependence regarding the coupler length.

Taking into account that the variation in the refractive index to change the coupler of the bar state to the cross state can be obtained by $\Delta \mathrm{nL}_{\mathrm{c}}=\lambda / 2[8]$, and as we have that $L_{c}=\frac{2 \pi}{\Delta \beta_{d}}$, we obtain that

$\Delta n=\frac{\lambda \Delta \beta_{d}}{4 \pi}$.

Taking into account that $\Delta \mathrm{n}(\mathrm{n}) \mathrm{nL}_{\mathrm{c}}=\lambda / 2$, we get $\Delta n\left({ }_{n}\right)=\frac{\lambda \Delta \beta_{d}}{4 \pi n}$.

Figure 8(a) shows a plot of $\Delta \mathrm{n}$ versus $\mathrm{L}_{\mathrm{c}}$ and Figure $8(\mathrm{~b})$ shows the graph of the required optical power of the command signal $(\mathrm{P})$, versus $\mathrm{L}_{\mathrm{c}}(\mu \mathrm{m})$.

We can note that the $\Delta \mathrm{n}$ and $\mathrm{P}$ dependence regarding the coupler length is very large.

The nonlinear effects are enhanced considerably in siliconon-insulator (SOI) waveguides because of the large values of the Kerr parameter and the Raman gain coefficient and the tight confinement of the optical mode. In practice, two-photon absorption (TPA) limits the extent of SPM through nonlinear absorption and generation of free carriers that not only absorb light (free-carrier absorption, or FCA) but also modify the refractive index (free-carrier dispersion, or FCD).

The FCD-induced phase shift has a sign opposite to that of the Kerr effect. This can be understood by noting that free carriers reduce the refractive index while the Kerr effect increases it. However, the TPA-induced free-carrier density $\left(\mathrm{N}_{\mathrm{c}}\right)$ is negligible when the input peak intensity $\mathrm{I}_{0}$ satisfies the condition $I_{0} \ll 3 h v_{0} / \sigma T_{0}$, where $T_{0}$ is the pulse width, and $\sigma$ is the FCA coefficient. For example, $\mathrm{I}_{0}$ should be $<<2.7$ $\mathrm{GW} / \mathrm{cm} 2$, for $\mathrm{T}_{0}=10 \mathrm{ps}$ [14].

To analyze the gradual increment of the refractive index using the PWE method, we gradually increase the dielectric constant of the coupling region, leaving unchanged the rest of the coupler structure. The results of these simulations show the effects associated with the gradual increase in the power of the command signal.
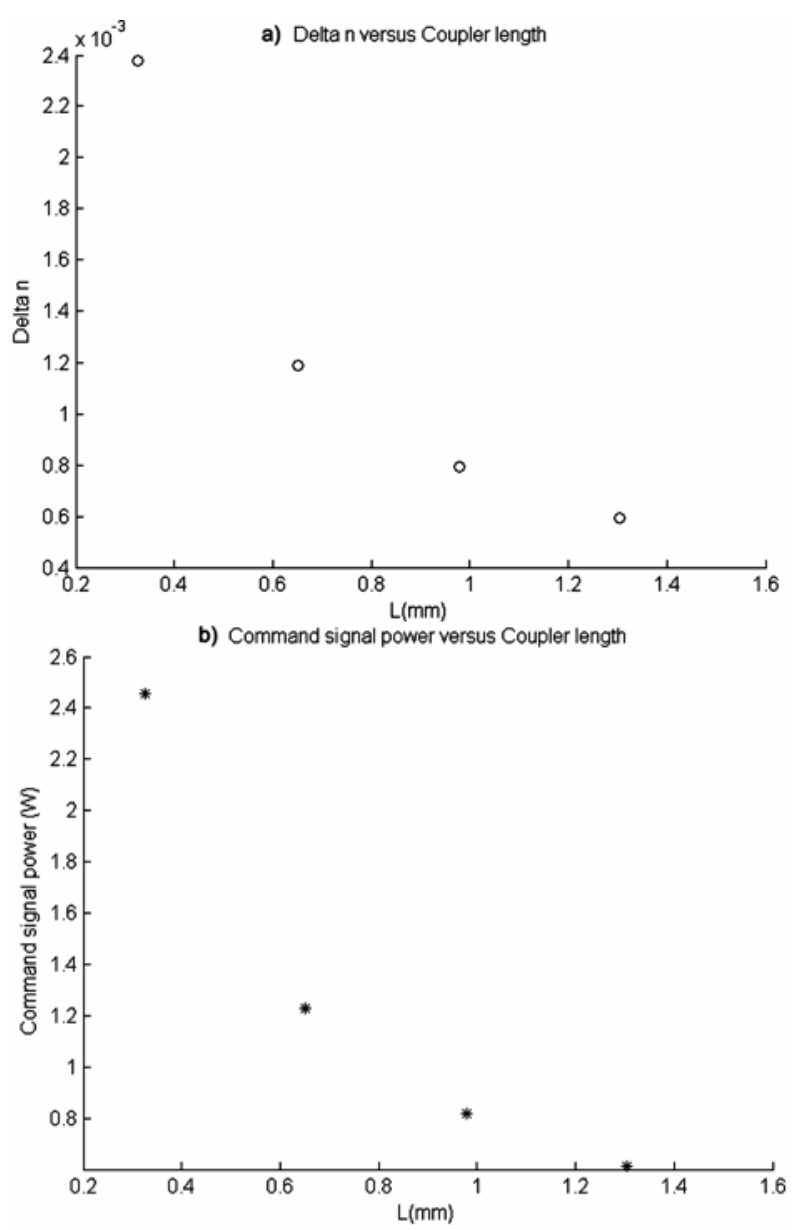

Fig. 8. $\Delta \mathrm{n}$ and $\mathrm{P}$ dependence regarding the coupler length.

The desired change in the refractive index depends on the optical power of the command signal. However, the change in refractive index depends on the data signal wavelength and on the coupler length. Figure 9 shows the graph of the optical power of the command signal vs. data signal wavelength for the minimal coupler length $(326 \mu \mathrm{m})$.

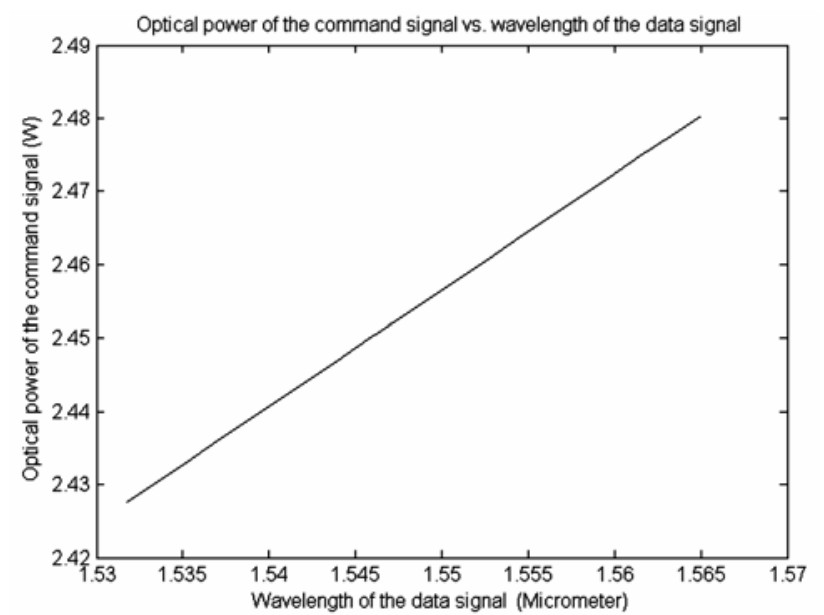

Fig. 9. Command signal power P vs. data signal wavelength (coupler length ten times greater than the minimal coupler length).

As expected, the required optical power of the command signal grows linearly with the increase of the wavelength of the data signal. 
The periodically corrugated interfaces of the PhCs waveguides can originate coupling between the co-directional fundamental mode and counter-propagation higher-order modes, leading to the formation of mini-stopbands (MSBs). Our simulations took into account the wave vectors within the PBG but outside of MSBs.

Direct coupling of the light sources to the narrow waveguides within the directional coupler (DCWGs) is not possible as this difference in the modal cross-sectional area results in high coupling loss. A serious problem arises because insufficient light coupled into PC devices will hinder their functionality and reliability. To solve this problem, several methods of coupling have been implemented to couple light efficiently to the DCWGs. For example, We can use a tapered waveguide (an elongated guiding structure for electromagnetic wave transmission that gradually becomes narrower toward one end), between de coupler waveguides and the external optical sources, to obtain low reflection loss, as well as higher coupling-efficiency improvement. Therefore, the main function of the tapered waveguide is to provide low loss connections between waveguides with different crosssectional areas [15].

We can use our coupler to obtain an all-optical switch with a crossbar wide-sense non-blocking space switch matrix, where any unused input port can be connected to any unused output port, without requiring any existing connection to be rerouted. [16].

An all-optical switch, which uses a space switch matrix can works in a full-optical WDM system [17 - 19]. Figure 10 shows a crossbar wide-sense non-blocking switch fabric. The $4 \times 4$ switch fabric consists of $162 \times 2$ switches. Indeed, the $2 \times 2$ switches can be the $2 \times 2$ directional couplers embedded in photonic crystal.

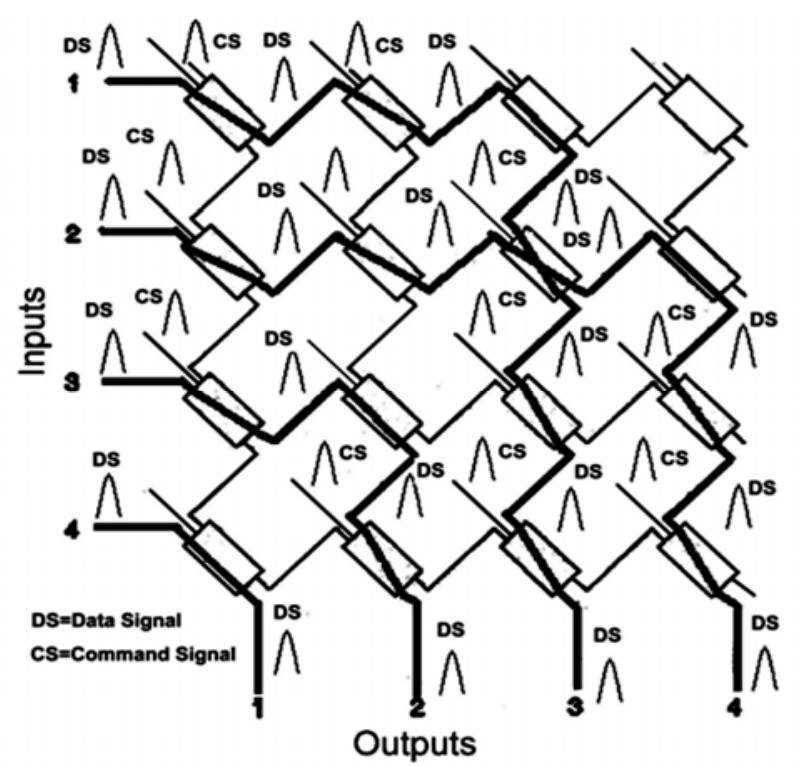

Fig. 10. $4 \times 4$ self-controlled crossbar switch fabric.

The interconnection between any input port and any output port is established as follows: The data signal travels through the couplers in row $\mathrm{i}$, until it reaches column $\mathrm{j}$, where it travels through the couplers in column $\mathrm{j}$, until it reaches the desired output port in colunn $\mathrm{j}$.

When desired, the command signal is inserted simultaneously with the data signal in the coupler. So this coupler works in the cross state, providing the switching of the signal.

\section{CONCLUSION}

We have proposed a directional coupler embedded in photonic crystal, which can acts by an external command signal $(\mathrm{u}=0.25)$. Therefore, this device can operate as switching cell in an all-optical switch for the $\mathrm{C}, \mathrm{L}$, and $\mathrm{U}$ bands of ITU.

To get low optical power for the command signal, we need low value of $\Delta n$, and/or high value of $n_{2}$. The desired change in the refractive index depends only on the wavelength of the command signal and on the length of the coupler. Hence, the optical power is dependent on the coupler length that was chosen. For example, if the coupler length is $326 \mu \mathrm{m}$ to the wavelength $\lambda=1,55 \mu \mathrm{m}$ the optical power need to be $2.46 \mathrm{~W}$. However, if the coupler length is $978 \mu \mathrm{m}$ to the wavelength $\lambda$ $=1,55 \mu \mathrm{m}$ the optical power must be just $0.82 \mathrm{~W}$. We can use similar reasoning for any wavelength used in the transmission system.

\section{ACKNOWLEDGMENT}

This work was partly sponsored by National Council of Scientific and Technological Development (Conselho Nacional de Desenvolvimento Científico e Tecnológico $\mathrm{CNPq}$ ).

\section{REFERENCES}

[1] J. Yonekura, M. Ikeda, and T. Baba, “Analysis of Finite 2-D Photonic Crystals of Columns and Lightwave Devices Using the Scattering Matrix Method", IEEE J. Lightwave Technol., 17 1500-1508, 1999.

[2] S. Boscolo, M. Midrio and C. G. Someda, "Coupling and Decoupling of Electromagnetic Waves in Parallel 2-D Photonic Crystal Waveguides", IEEE J. Quantum Electron., 38 47-53, 2002.

[3] A. Martinez, F. Cuesta, and J. Marti, "Ultrashort 2-D Photonic Crystal Directional Couplers", IEEE Photonics Technl. Lett., 15 694-696, 2003.

[4] Y. Tanaka, H. Nakamura, Y. Sugimito, N. Ikeda, K. Asakawa, and K. Inoue, "Coupling Properties in a 2-D Photonic Crystal Slab Directional Coupler With a Triangular Lattice of Air Holes", IEEE J. Quantum Electron. 41, 76-84, 2005.

[5] Govind P. Agrawal, "Nonlinear fiber optics", Academic Press, 295 301, 414, 1995.

[6] John D. Joannopoulos, Steven G. Johnson, Joshua N. Winn, and Robert D. Meade, "Photonic Crystals, Molding the Flow of Light", Princeton University Press, 66 - 93, 2008.

[7] Noritsugu Yamamoto, Toru Ogawa, and Kazuhiro Komori, "Photonic crystal directional coupler switch with small switching length and wide bandwidth", Optics Express, Vol. 14, No. 3, pp 1223 - 1229, 2006.

[8] D. M. Beggs, T. P. White, L. O'Faolain and T. F. Krauss, "Design and fabrication of a photonic crystal directional coupler for use as an optical switch", School of Physics and Astronomy, University of St Andrews, FrC3, ECIO '08, 2008.

[9] S.G. Johnson, and D. Joannoopoulus, "Block-iterative frequency domain methods for Maxwell equations in planis basis", Opt. Express 8, $173-$ 190, 2001.

[10] Ahmed Sharkawy, Shouyuan Shi and Dennis W. Prather, "Electrooptical switching using coupled photonic crystal waveguides", Optics Express 1048, 2002. 
[11] Pi-Gang Luan, Kao-Der Chang, "Transmission characteristics of finite periodic dielectric waveguides", Optical Society of America, 2006.

[12] Alan D. Bristow, Nir Rotenberg, and Henry M. van Driel, "Two-photon absorption and Kerr coefficients of silicon for 850-2200 nm", Applied Physics Letters 90, 2007.

[13] F. Cuesta-Soto, A. Martinez, J. Garcia, F. Ramos, P. Sanchis, J. Blasco, and J. Marti, "All-optical switching structure based on a photonic crystal directional coupler", Optics Express 161, 2004.

[14] Lianghong Yin and Govind P. Agrawal, "Impact of two-photon absorption on self-phase modulation in silicon waveguides", Optics Letters, Vol. 32, No. 14, pp 2031 - 2033, 2007.

[15] E. H. Khoo, A. Q. Liu, and J. H. Wu, "Nonuniform photonic crystal taper for high-efficiency mode coupling", Optics express, Vol. 13, No. 20, pp. $7748-7759,2005$.

[16] Papadimitriu, Chrisoula Papazoglou, and Andreas S. Pomportsis, "Optical Switching: Switch Fabrics, Techniques, and Architectures", Journal of Lightwave Technology, Vol. 21, 384 - 390, 2003.

[17] S. L. Danielsen, B. Mikkelsen, C. Joerggensen, T. Durhuus, and K.E Sutbkjaer, "WDM packet switch architectures and analysis of the influence of tuneable wavelength converters on the performance", J. Light-wave Technol., Vol. 15, 219 - 227, 1997.

[18] S. Yao, B. Mukheree, and S. Dixit, "Advances in photonic packet switching: An overview”, IEEE Commun., 84 -94, 2000.

[19] A. Rodriguez-Meral, P. Bonenfant, S. Baroni, and R. Wu, "Optical data networking: Protocols, Technologies, and arquitectures for next generation optical transport networks and optical intenetworks", J. Lightwave Technol., Vol. 18, $1885-1870,2000$.

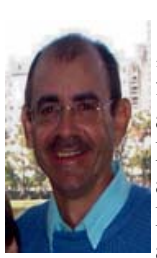

Antonio Sergio Bezerra Sombra was born in Jaguarauana ,CE ,Brazil in 1959. He received the BSc and MSc degree in Physics from Federal University of Ceará in 1981 and 1984, respectively, and the $\mathrm{PhD}$ from the Federal University of Pernambuco in 1990.

$\mathrm{He}$ is head of the Telecommunications and Materials Science and Engineering Laboratory (LOCEM) at the Physics Department at Federal University of Ceara. The main interest areas includes research and development in optical fiber and planar devices for optical networks. They are also involved in the study of electric, dielectric and piezoelectric properties of new ceramics and films for microwave and radio-frequency applications.

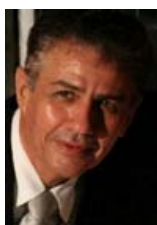

Almir Wirth Lima Junior is Telecommunications Engineer, with an Advanced Training Course on Telecommunications by Telekom in Germany, Master and Doctor on Teleinformatics by Federal University of Ceará (U.F.C.). The Authors' activities are: Consulting Services in System of Telecommunications, as well as Teaching in University Courses on Telecommunications and Computers Networks (more than twenty years of experience as University Professor). The author has worked 10 (ten) years in Telecommunications of Brazil (TELEBRAS) in the areas of Switching, Transmission and Computers Networks, where has taught several courses on Telecommunications and Computers' Networks. The Authorship of several books are also included in the Curriculum Vitae of the Author, including "Networks Technologies \& Data Communications," Modern Telecommunications", "Multimedia Telecommunications," "Internet and Computers Networks", "All About Optical Fibers - Theory \& Practice", "ISDN", "Basics of Electricity and Electronics", "PCs Hardware", "MSProject" and "AutoCAD". Finally, the Author wrote several manuscripts, which were published in scientific journals and international conferences. 